\title{
Left Ventricle Volume Estimation From 3D SPECT Reconstruction
}

\author{
O. Garcia-Panyella ${ }^{1}$, A. Susin $^{2}$ \\ ${ }^{1}$ La Salle School of Engineering, ${ }^{2}$ Technical University of Catalonia, Barcelona, Spain
}

\begin{abstract}
Our approach describes the three-dimensional reconstruction of the internal and external surfaces of the human's left ventricle from actual SPECT data.

The reconstruction is a first process fitting in a complete VR application that will serve as an important diagnosis tool for hospitals.

Beginning with the surfaces reconstruction, the application will provide volume and interactive real-time manipulation with the model. We focus on speed, precision and smoothness for the final surfaces.

We present several recoveries with non-missing data and partial missing data, performed using different fillings for a PHANTOM test volume. We also present a reconstructed cardiac cycle from an actual patient data, all along with its associated ejection fraction estimation.
\end{abstract}

\section{Introduction}

Access to a 3D model obtained from patient's data can have several applications like support on diagnosis, surgery planning, student's training or even remoteoperation.

A first approximation to the problem would be using a manual process with specific image-processing software though it would require deep medical knowledge and experience.

Some parts of the human body like skin or bones have clear intensity-gradient variations that make surfacereconstruction techniques, like the Marching Cubes, suitable. Unfortunately these techniques don't work when regions are formed from soft tissue. That's the case of the heart, liver or muscles.

In order to solve those cases several contour-based techniques have been introduced like Snakes [1], Active Nets, 3D Active Nets and Active Cubes. In all the approximations the evolution is similar, an initial parametrically defined contour or mesh which deforms attracted to a certain energy minima, numerically implemented as a finite-differences method.

Other approaches include the FEM method [2]. Those methods give nice results but are computationally expensive and complex.
The dynamic model that we present solves the same type of problems using the evolution of a deformable mesh affected by internal and external forces. Internal forces are defined in terms of elasticity; external forces are derived from the data set as a vector field called GVF [3]. The use of the GVF vector field avoids some of the limitations of traditional snakes related to initial distance to data and robustness in concave regions.

\section{Input imagery}

The system takes as its input SPECT (Single Photon Emission Computed Tomography) images. Those images give functionality keys about the organ and do not describe its anatomy. From this knowledge it becomes clear that isquemic areas, with poor or absence of blood irrigation, won't be shown in the images.

For the left ventricle, typically acquisition procedures involve 64 planes normal to the symmetry axis (X and $\mathrm{Y}$ ) and 24 planes along it $(\mathrm{Z})$. Final data results on lowresolution images attending to its spatial resolution $(3 \mathrm{~mm}$ $\mathrm{x} 3 \mathrm{~mm} \times 5 \mathrm{~mm}$ ). That will be a big issue, especially when recovering the internal surface due to its lack of resolution.

Moreover if we are dealing with a pathological organ, we will obtain data absence in terms of "holes" in the images that will difficult the surface recovering.

\section{Evolution scheme}

The evolution scheme is based on Newtonian dynamics where the model is built from particles that move due to internal and external forces.

Figure 1 shows the Newtonian dynamics law:

$$
F_{i}=m_{i} \cdot \ddot{x}_{i} \Rightarrow\left\{\begin{array}{c}
\dot{x}=v \\
\dot{v}=F_{i} / m_{i}
\end{array}\right.
$$

Figure 1 . Newtonian dynamics law for the $i_{\text {th }}$ particle.

Where $\mathrm{F}$ stands for force, $\mathrm{m}$ for mass, $\mathrm{x}$ for position and its successive derivatives for velocity and acceleration respectively.

In order to solve the system, we need a numerical 
method. For our tests, we apply an explicit method, a Runge-Kutta 4 solver. Reports on these can be found at $[4,5]$.

\subsection{Internal forces}

We define four possibilities for our model depending on the applied elasticity forces. Those are the Plane deformation model, the Spring-Mass deformation model, the Restricted Spring-Mass deformation model and the Free deformation model.

In fact, all the results that we present have been achieved by the Free deformation model, where the only existing force is the external one, derived from the dataset. There's no connectivity between particles and topology must be maintained using a smoothing algorithm, apart from the evolution scheme. All the models have been extensively reported in $[4,5]$.

\subsection{External forces}

External forces consist on the minimization of a functional that mixes the information derived from the image-intensities gradient with a diffusion term that allows the field to be spread out.

The vector field that acts as the external force is obtained then by minimizing the functional depicted in figure 2 :

$$
\varepsilon=\iiint \mu \sum_{\mathrm{i} \in\{\mathrm{x}, \mathrm{y}, \mathrm{z}\}}\left(\mathrm{u}_{\mathrm{i}}^{2}+\mathrm{v}_{\mathrm{i}}^{2}+\mathrm{w}_{\mathrm{i}}^{2}\right)+|\nabla \mathrm{I}|^{2}|\mathrm{~V}-\nabla \mathrm{I}|^{2} \mathrm{dxdydz}
$$

Figure 2. The energy-based functional.

This functional consists on two well-differentiated terms. On the left, the diffusion term that spreads the field when variations on intensities are negligible. On the right the property term, that dominates the expression when variations are important.

I stands for the property value; $\mathrm{u}, \mathrm{v}, \mathrm{w}$ are the vector field components; the $\mu$ parameter will control the balance between both terms. Details on its resolution can be found at [3].

\section{The Phantom dataset}

In order to measure the reliability of the system from the results point of view, it becomes necessary to test a dataset with known volume.

The phantom is a test-model that offers known geometry and volume, allowing us to compare with the obtained results.

\subsection{The Vall Hebrón hospital phantom}

As a first experiment, we have used the Vall Hebrón Hospital phantom, which is presented in figure 3 :

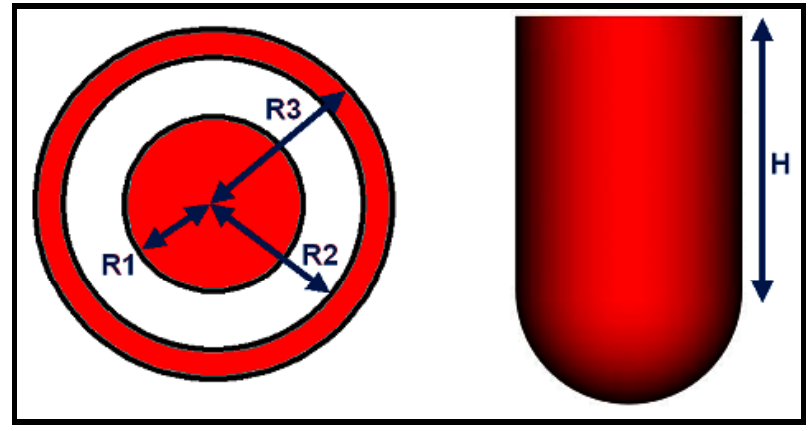

Figure 3. The Vall Hebrón Hospital Phantom dataset.

With R1 $=20 \mathrm{~mm}, \quad \mathrm{R} 2=35 \mathrm{~mm}, \quad \mathrm{R} 3=45 \mathrm{~mm}$ and $\mathrm{H}=55 \mathrm{~mm}$. Those distances give external and internal theoretical volumes of $305301.4 \mathrm{~mm}^{3}$ and $89794 \mathrm{~mm}^{3}$ respectively.

In order to compute the real volume, we filter several data voxels, rejecting those with intensities lower than $20 \%$ of the maximum. Final data is showed in figure 4 :

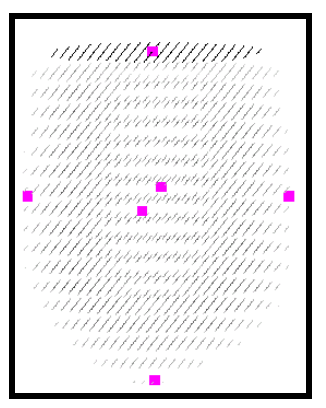

Figure 4. The filtered phantom dataset.

If we quantify the data volume we obtain a value of $368782 \mathrm{~mm} 3$. This volume differs from the theoretical one in a $12 \%$.

We must take into account the low resolution of the data and the error due to the quality of the reception from the medical equipment.

Figure 5 shows several simulations performed using explicit methods. From top to bottom the Euler, Midpoint and Runge-Kutta- 4 methods. The third column shows the computed $\%$ of error between the recovered surface volume and the filtered data.

All three numerical schemes are satisfactory in the sense that achieve errors less than $2 \%$.

In figure 5, flat shading stands for triangle-based shading and Gouraud shading for vertex-based shading. Both are computer graphics techniques.

Time is expressed in seconds and it is referred to the simulation (reconstruction) process. 


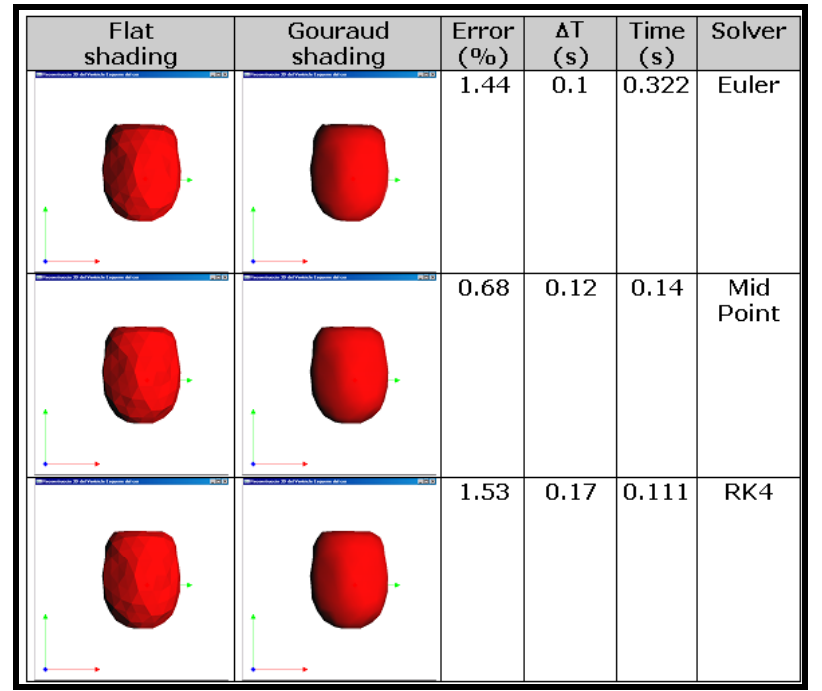

Figure 5. Evaluation of the phantom volume from the reconstructed external surface.

\subsection{The Mayo clinic phantom}

In order to evaluate recovering results with partial missing data, we require using different fillings of the phantom volume. We present some recovering test experiments with $10 \%, 32 \%$ and $53 \%$ percentages of missing volume data, always referred to the $100 \%$ of the total.

In these test, a different phantom model (more geometric heart-like) was used. The Mayo clinic phantom. Its total volume is $265501 \mathrm{~mm}^{3}$.

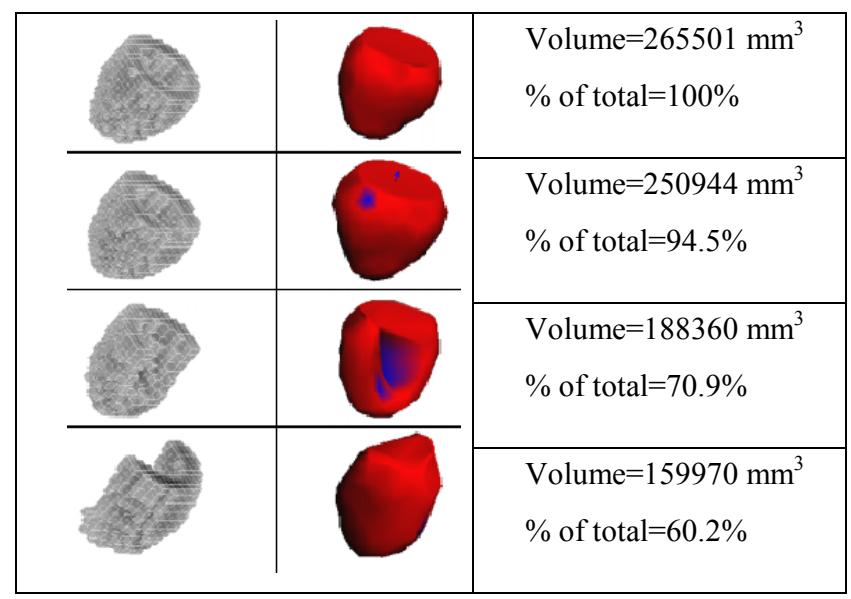

Figure 6. Recovered surfaces from partial data.

Here the first column shows the initial data to be recovered, the second column depicts the final meshes and the third column points out the recovered final volumes (absolute values and percentage of total).

As we can see, the best recovering is the third one because it gives a percentage of missing volume of $29.1 \%$ against the $32 \%$ of the real data emptied. This represents a relative percentage error of 0.09 . For the other test examples we obtain relative errors of 0.45 and 0.24 respectively.

\section{Reconstruction process}

Figure 7 shows the complete process for a reconstruction, in this case an actual left ventricle (the first dataset of a cardiac cycle). Both, the external (left) and internal (right) surfaces are recovered starting with an initial bounding ellipsoid:

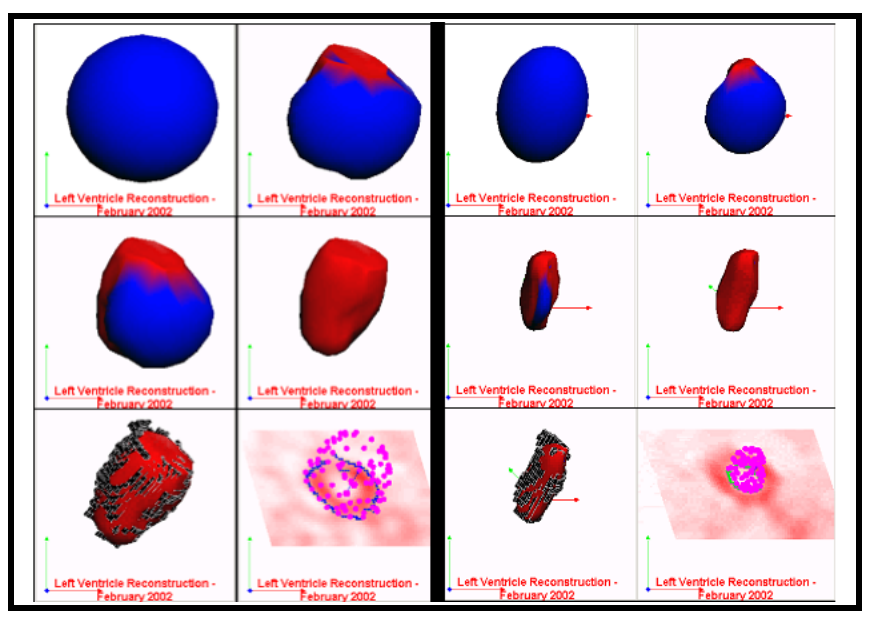

Figure 7. A reconstruction process.

The sequence starts with the initial meshes. When the reconstruction begins, the meshes change their color as long as the particles reach the marked voxels (borders). Finally the figure shows the border voxels (black cubes) superimposed to the meshes and the particles over data slices.

\section{Ejection Fraction}

We present a reconstructed cardiac cycle (see figure 8) from an actual patient data, all along with its associated ejection fraction (EF) estimation. EF value is estimated using medical software and compared with our system evaluation.

\subsection{Complete cardiac cycle}

Figure 8 shows the cycle, formed by eight temporal acquisitions. Each data set consists on $64 \times 64 \times 24$ voxels, with spatial resolutions of $2.87 \mathrm{~mm}(\mathrm{X}), 2.87 \mathrm{~mm}$ (Y) and $5.74 \mathrm{~mm}(\mathrm{Z})$.

Table 1 shows the extracted volumes $\left(\mathrm{mm}^{3}\right)$ that will allow us to compute the EF. Volumes are evaluated for every mesh. 


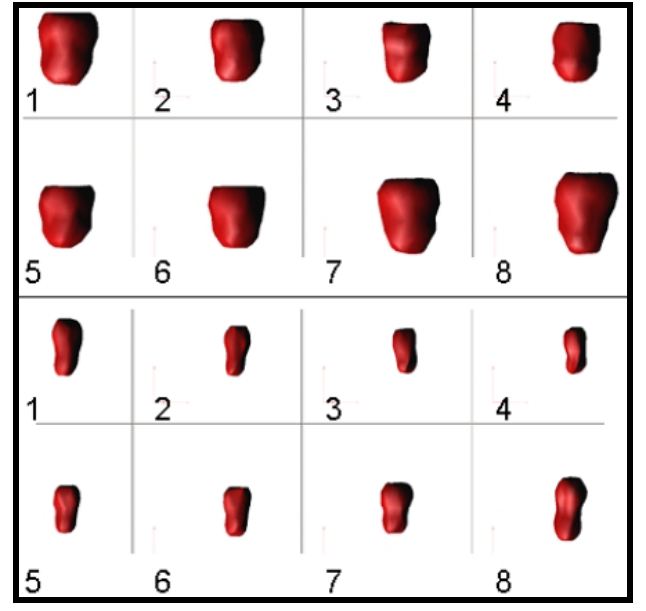

Figure 8. Complete cardiac cycle with external surfaces (top) and internal surfaces (bottom).

Table 1. Obtained volumes for the eight meshes.

\begin{tabular}{|l|c|c|c|c|}
\hline & $\mathbf{1}$ & $\mathbf{2}$ & $\mathbf{3}$ & $\mathbf{4}$ \\
\hline Int. & 74741 & 45677 & 36523 & 32956 \\
\hline Ext. & 443762 & 316804 & 254112 & 250131 \\
\hline Wall & 369021 & 271127 & 217589 & 217175 \\
\hline & $\mathbf{5}$ & $\mathbf{6}$ & $\mathbf{7}$ & $\mathbf{8}$ \\
\hline Int. & 47237 & 55825 & 63133 & 79581 \\
\hline Ext. & 299779 & 345830 & 451525 & 473608 \\
\hline Wall & 252542 & 290005 & 388392 & 394027 \\
\hline
\end{tabular}

\subsection{Ejection fraction estimation}

Using the values presented in table 1, an EF estimation can be calculated for this ventricle.

$$
\begin{aligned}
& E F=\frac{\text { DiastoleEndVolume }- \text { SystoleEndVolume }}{\text { DiastoleEndVolume }}= \\
& =\frac{79581-32956}{79581}=0.586 \Rightarrow 58.6 \%
\end{aligned}
$$

Figure 9. EF calculation.

Here the volumes are internal. As it is showed, EF value is inside the interval $50 \%-70 \%$ which states for a non-pathological situation.

Medical software derived an EF value of $53 \%$, which stands for a percentage error of $5.6 \%$ with regard to our application.

\section{Nuclear ventriculography}

We noted that in some pathological cases, it can be interesting to use data from nuclear ventriculography instead of using standard perfussion imagery. If the volume of isquemic tissue is really severe, the detection of the inner borders can be better achieved by this way.

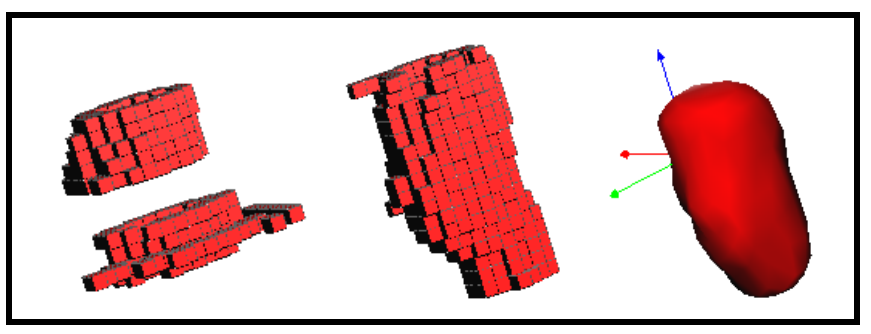

Figure 10. Perfussion borders (left); Ventriculography borders (middle); Final mesh (right).

As shown in figure 10 for the internal surface, data borders detected from the perfussion data present an important lack of consistency. If we take a look at the same test done over the ventriculography, the borders are much better labelled. Then the final surface can be perfectly recovered.

\section{Acknowledgements}

The authors wish to thank all the members of the medical applications research group at the IRI-UPC, the Nuclear Cardiology team at Vall Hebrón Hospitals and the people integrating the graphics \& VR group at the CITeM.

This work has been partially financed by the TIC20001009 project. The first author is granted by an EPSON "Rosina Ribalta" prize.

\section{References}

[1] Kass M, Witkin A, Terzopoulos D. Snakes: active contour models. International Journal of Computer Vision 1988;1:321-331.

[2] Cohen LD, Cohen I. Finite-element methods for active contour models and balloons for 2-D and 3-D. IEEE Transactions PAMI 1993;15(11):1131-1147.

[3] Xu C, Prince JL. Snakes, shapes, and gradient vector flow. IEEE Transactions on Image Processing 1998;7(3):359369.

[4] Susin A, Garcia O. Modelo dinámico para la reconstrucción del corazón humano. XVII CEDYA, VII Congreso de Matemática Aplicada 2001;1:733-734.

[5] Garcia O, Susin A. Left ventricle's surface reconstruction and volume estimation. Terceres jornades de recerca en Enginyeria Biomèdica 2002;1:1-10.

Address for correspondence.

${ }^{1}$ La Salle School of Engineering.

C/ Quatre Camins,2. 08022 Barcelona, Spain, Europe. oscarg@salleURL.edu - http://www.salleurl.edu/ oscarg/

${ }^{2}$ Departament Matematica Aplicada.

Av/ Diagonal, 647. 08028 Barcelona, Spain, Europe. toni.susin@upc.es - http://www-mal.upc.es/ susin/ 